J. Lake Sci. (湖泊科学), 2020, 32(6): 1827-1836

DOI 10. 18307/2020. 0622

(c) 2020 by Journal of Lake Sciences

\title{
波浪条件下刚性植被茎干紊流对沉积物再悬浮的促进作用”
}

\author{
易雨君 ${ }^{1 * *}$, 唐彩红 ${ }^{1}$, 张尚弘 ${ }^{2}$ \\ (1: 北京师范大学水环境模拟国家重点实验室, 北京师范大学环境学院, 北京 100875) \\ ( 2 : 华北电力大学水利与水电工程学院, 北京 102206$)$
}

\begin{abstract}
摘 要: 浅水湖泊沉积物再悬浮过程促进了沉积物内源性物质的释放,造成湖泊水体内源污染, 加剧湖泊水质污染与生 态恶化. 开展刚性植被不同分布格局下的沉积物再悬浮水槽试验, 能为浅水草型湖泊、滨海湿地等水环境治理与生态修 复提供理论参考. 本研究通过开展实验室波浪水槽试验, 检验了 3 种直径 12 种密度的刚性模型植被对波浪扰动白洋淀沉 积物再悬浮的影响, 通过测定不同植被情景不同波浪条件下的瞬时速度和沉积物再悬浮浓度, 详细阐述了植被产生的紊 动能对沉积物再悬浮临界状态的影响, 构建并验证了沉积物再悬浮植被紊流模型, 预测了沉积物再悬浮的临界速度, 为 湿地生态恢复与保护提供参考. 结果表明:1) 植被的存在能有效增加近床面的紊动能;2) 紊动能是控制沉积物再悬浮的 关键性因素;3) 沉积物再悬浮的临界波速与植被的固相体积分数相关.
\end{abstract}

关键词: 刚性植被;沉积物再悬浮;波浪;紊动能;临界阈值;浅水湖泊

\section{Effect of stem-generated turbulence on sediment resuspension within rigid vegetation canopies in waves*}

\author{
YI Yujun $^{1 * *}$ TANG Caihong $^{1} \&$ ZHANG Shanghong ${ }^{2}$ \\ (1: State Key Laboratory of Water Environment Simulation, School of Environment, Beijing Normal University, Beijing \\ 100875, P.R.China) \\ (2: School of Water Resources and Hydropower Engineering, North China Electric Power University, Beijing 102206, P.R. \\ China)
}

\begin{abstract}
Sediment resuspension in shallow lakes promotes the nutrients release, which leads to the internal pollution, degrading the water quality and lake ecology. This research aims to study the impact of rigid vegetation on wave-driven sediment resuspension through wave flume experiments. It could provide a theoretical reference for environmental management and ecological restoration of shallow lakes and coastal wetlands. Vegetation canopies were constructed by rigid cylinders considering three diameters and 12 vegetation densities. The near-bed instantaneous velocity was measured within vegetation canopies and under different wave conditions by a Nortek Vectrino at sampling rate of $200 \mathrm{~Hz}$. Suspended sediment concentrations were measured using an optical backscatter with frequency of $20 \mathrm{~Hz}$. The vegetation-generated turbulence was positively linear with the root mean square of wave velocity. This vegetated turbulence increased with an increasing ratio $\left(A_{w} / S\right)$ of wave excursion $\left(A_{w}\right)$ to stem spacing $(S)$ when $A_{w} / S>1$, and was similar with bare bed case when $A_{w} / S<1$. The concentration of sediment resuspension increased with growing solid volume fraction. The critical state of resuspension was initiated when the suspended sediment concentration exceeded the background level. Higher solid volume fraction generated higher turbulence, which promoted a small critical wave velocity. A vegetation-generated turbulence model for sediment resuspension was proposed and validated using the measured turbulence in the model canopy. Therefore, we confirmed that the magnitude of stem-generated turbulence is a function of solid volume fraction. This model proved the key role of turbulent kinetic energy to control the initial sediment resuspension. Based on this, a threshold model of critical velocity for sediment resuspension was proposed and validated. It could predict the critical near-bed wave velocity for sediment resuspension
\end{abstract}

* 2019-12-27 收稿;2020-04-01 收修改稿.

国家重点研发计划项目 (2018YFC0407403)、国家自然科学基金项目 (51722901) 和北京市自然科学基金项目 (JQ19034) 联合资助.

** 通信作者; E-mail: yiyujun@ bnu.edu.cn. 
within rigid vegetation canopy or sheath with diameters of $0.32 \mathrm{~cm}$ to $1.2 \mathrm{~cm}$. The applicable particle size was limited to $85-$ $280 \mu \mathrm{m}$.

Keywords: Rigid vegetation; sediment resuspension; wave; turbulence; velocity threshold; shallow lakes

水生植被广泛分布于河流、湖泊以及滨海湿地等浅水域中, 为鱼类、底栖动物和其他水生动物提供食物 和栖息地 ${ }^{[-3]}$. 水生植被可以吸收水体过剩营养物质,净化水质改善透明度; 通过植物根系稳定河床保护河 岸; 还可衰减波浪, 避免海岸带遭受侵蚀 ${ }^{[4-5]}$. 在浅水域, 沉积物易受风生波影响导致再悬浮, 加剧悬浮颗粒 中营养物和污染物的释放, 从而导致水体营养物质的增加以及水质恶化 ${ }^{[6]}$. 太湖的原位观测实验表明, 太湖 底泥沉积物再悬浮的临界剪切应力为 $0.03 \sim 0.04 \mathrm{~N} / \mathrm{m}^{2}$, 风生浪是导致太湖底泥沉积物再悬浮的主要驱动 力, 当风速达到 $6.5 \mathrm{~m} / \mathrm{s}$ 时, 沉积物再悬浮含量以及水体氮磷营养物质浓度会急剧增加 ${ }^{[6]}$. 一方面, 水生植被 可以有效削减波浪并降低近床面波速 ${ }^{[7-8]}$, 从而减少波浪驱动的沉积物再悬浮 ${ }^{[9]}$; 另一方面, 有研究表明波 浪与植被的相互作用能增强近床面的紊流 ${ }^{[10-11]}$, 而紊流的增加能增强沉积物的再悬浮作用. 研究发现, 柔性 沉水植被的刚性叶鞘具有与挺水植被类似的性质,刚性叶鞘部分产生的近床面紊动能与波浪轨道运动半径 $\left(A_{w}\right)$ 和植株间距 $(S)$ 的比值 $\left(A_{w} / S\right)$ 有关, 当 $A_{w} / S>0.5$ 时, 相比无植被情景, 植被能有效增加近床面紊动 能 ${ }^{[1]}$. 随机排列的红树林模型植被实验结果表明, 波浪驱动的沉积物悬浮浓度与紊流强度之间存在正相关 关系, 再悬浮的临界波速在植被层内相对于光滑床面更低, 其原因在于植被产生的紊流作用促进了沉积物

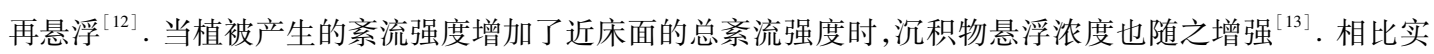
验室研究, 田间尺度上密集的鳗草冠层内波浪速度和紊流的减少, 反过来减少了沉积物再悬浮 ${ }^{[14]}$. 同样, 在 浅水湖泊中, 与无植被区域相比, 沉水和挺水植物的存在使滇池福宝湾生态恢复区的底泥再悬浮量、氮磷释 放量均削减了 $80 \%$ 以上 ${ }^{[9]}$. 田间观测和实验室观测之间互相矛盾的结果反映了植被对波浪衰减 ${ }^{[15]}$ 和紊流 形成的双重影响 ${ }^{[11]}$. 即是植被产生的紊流可以局部增强沉积物再悬浮作用 ${ }^{[12-13]}$, 但是大面积植被的波浪衰 减可以有效减少沉积物的再悬浮 ${ }^{[14]}$. 本研究以植被茎干产生的紊流可局部增强沉积物再悬浮为切人点, 旨 在阐明波浪条件下刚性植被茎干产生的紊流如何影响沉积物再悬浮过程, 提出沉积物再悬浮植被紊流模 型, 基于该模型预测沉积物再悬浮的临界波速, 通过水槽试验的实测数据对该模型进行验证, 并将其扩展到 其他研究中.

\section{1 植被紊流与临界波速的理论关系}

植被产生的紊流浴旋将能量从平均流场转移到紊流中, 单向水流中非淹没刚性植被产生的紊流模型 $k_{t v}$ 可表示为 ${ }^{[16]}$ :

$$
k_{t v}=\delta^{2}\left(C_{D} \frac{2 \phi}{\pi(1-\phi)}\right)^{2 / 3} U^{2}
$$

式中, $U$ 为空间和时间平均的纵向速度, $C_{D}$ 为植被拖曳力系数, $\phi$ 是植被固相体积分数, $\delta$ 为比例因子. 对圆 柱形植被茎干而言, $\phi=m \pi d^{2} / 4$, 其中 $m$ 为植被茎干密度 (根 $\left./ \mathrm{m}^{2}\right), d$ 为植被直径. 公式 (1) 适用于 $d / S<$ 0.56 的植被情景.

研究表明, 近床面总紊动能 $\left(k_{t}\right)$ 可表示为植被产生的紊动能 $\left(k_{t v}\right)$ 与床面产生的紊动能 $\left(k_{t b}\right)$ 之和, 即是 $k_{t}=k_{t v}+k_{t b}{ }^{[17]}$. 在光滑床面的单向流动中, $k_{t b}$ 与水流产生的床面剪切应力 $\tau_{c}$ 之间存在线性关系, 已有研究 证实光滑床面 $\tau_{c} / \rho k_{t b}=0.19^{[18]}$. 此外, 床面剪切应力可以根据流速 $U$ 和床面摩擦系数 $C_{f}$ 估算, 即 $\tau_{c}=$ $\rho C_{f} U^{2}$, 使得 $k_{t b}=C_{b} U^{2}$, 其中 $C_{b} \approx C_{f} / 0.2$. 结合公式 (1), 单向流中近床面总紊动能可表示为:

$$
k_{t}=\delta^{2}\left(C_{D} \frac{2 \phi}{\pi(1-\phi)}\right)^{2 / 3} U^{2}+C_{b} U^{2}
$$

渠道内的流速结构或床面形态产生的紊流可以增强沉积物运动 ${ }^{[19]}$, 植被产生的紊流也具有类似的影 响. 单向流中沉积物的初始运动由紊动能的临界值决定, 根据公式 (2), 光滑床面的临界总紊动能 $k_{t c}=$ $C_{b} U_{c o}^{2}$, 式中, $U_{c o}$ 为光滑床面沉积物颗粒运动的临界速度 ${ }^{[17]}$. 对于临界状态而言, 沉积物再悬浮所需的临界 紊动能 $k_{t c}$ 为常数, 即是光滑床面的临界紊动能等于有植被时近床面的临界总紊动能. 等同于公式 (2)对临 
界紊流水平的定义,变换公式 $(2)$ 可得植被影响下沉积物运动的临界速度 $U_{c}$ :

$$
\frac{U_{c}}{U_{c o}}=\frac{1}{\sqrt{1+\frac{\delta^{2}}{C_{b}}\left(\frac{2 C_{D}}{\pi}\right)^{2 / 3}\left(\frac{\phi}{1-\phi}\right)^{2 / 3}}}
$$

对于单向流, 沉积物运动的临界速度随植被固相体积分数 $(\phi)$ 的增加而减小. Tinoco 和 Coco ${ }^{[12]}$ 结合 Yang 等 ${ }^{[17]}$ 的研究结果验证了沉积物输运模型 (公式 (3)), 用单向流的 Shields 数描述波浪驱动的沉积物再 悬浮过程, 用波周期内临界波速最大值 (即轨道波速 $U_{w}$ ) 代替临界流速, 以描述无植被 $\left(U_{w, c o}\right)$ 和植被 $\left(U_{w, c}\right)$ 情景下的沉积物再悬浮临界速度. 假设波浪条件下光滑床面产生的紊流 $\left(k_{t b, w}\right)$ 与床面切应力 $\tau_{w}$ 也呈线性关 系, Green and $\mathrm{Coco}^{[20]}$ 已证实 $\tau_{w}$ 与波速平方 $U_{w}^{2}$ 呈正比 $\left(\tau_{w}=\rho \cdot f_{w} \cdot U_{w}^{2} / 2\right), f_{w}$ 为波浪摩擦因子. 因此, 波浪条件 下光滑床面产生的紊动能 $k_{t b, w}=C_{b, w} U_{w}^{2}$ 合理.

将公式(2)中的流速替换为波速, 可得波浪驱动下的植被紊流方程为:

$$
k_{t w}=\delta^{2}\left(C_{D} \frac{2 \phi}{\pi(1-\phi)}\right)^{2 / 3} U_{w}^{2}+C_{b, w} U_{w}^{2}
$$

本研究通过水槽试验, 验证了公式 (4) 作为波浪条件下不同密度和直径的刚性植被层近床面紊流模型. 假设波浪条件下的沉积物再悬浮紊流临界水平 $k_{t c}$ 可以从光滑床面推断而来, 即 $k_{t c}=C_{b, w} U_{w, c o}^{2}$, 则通过公式 (4) 可得圆柱形植被层内波浪驱动的沉积物再悬浮临界波速为:

$$
\frac{U_{w, c}}{U_{w, c o}}=\frac{1}{\sqrt{1+\frac{\delta^{2}}{C_{b, w}}\left(\frac{2 C_{D}}{\pi}\right)^{2 / 3}\left(\frac{\phi}{1-\phi}\right)^{2 / 3}}}
$$

\section{2 实验设计与方法}

根据 2016 年对白洋淀春、夏、秋 3 个季节 12 个采样点的水生植被野外采样 (图 1a), S1 S12 分别为东 关码头、妴鹩岛、淀头、端村、采蒲台、圈头、苯林庄、光淀张庄、王家寨、大口子、烧车淀以及旅游码头. 结果显 示, 白洋淀挺水植被以芦苇和香蒲为主, 芦苇株高为 $2.70 \pm 0.33 \mathrm{~m}$, 株茎为 $0.77 \pm 0.15 \mathrm{~cm}$, 沉水植被以金鱼藻、 菹草、眼子菜科为优势种. 同时采集 12 个采样点的沉积物, 将其带回实验室物理风干后用激光粒度分析仪 (Microtrac S3500 Particle Size Analyzer)进行粒径测量. 结果表明, 白洋淀 12 个采样点的沉积物平均中值粒 径 $D_{50}=0.076 \mathrm{~cm}$, 属于细砂, 各采样点的沉积物粒径分布见图 $1 \mathrm{~b}$. 相比较而言, 白洋淀沉积物粒径大于太湖 $\left(D_{50}=0.009 \mathrm{~mm}\right)$ 、龙感湖 $\left(D_{50}=0.007 \mathrm{~mm}\right)$ 以及巢湖的湖岸 $\left(D_{50}=0.007 \mathrm{~mm}\right)$ 和湖中心 $\left(D_{50}=0.010 \mathrm{~mm}\right)^{[21]}$.
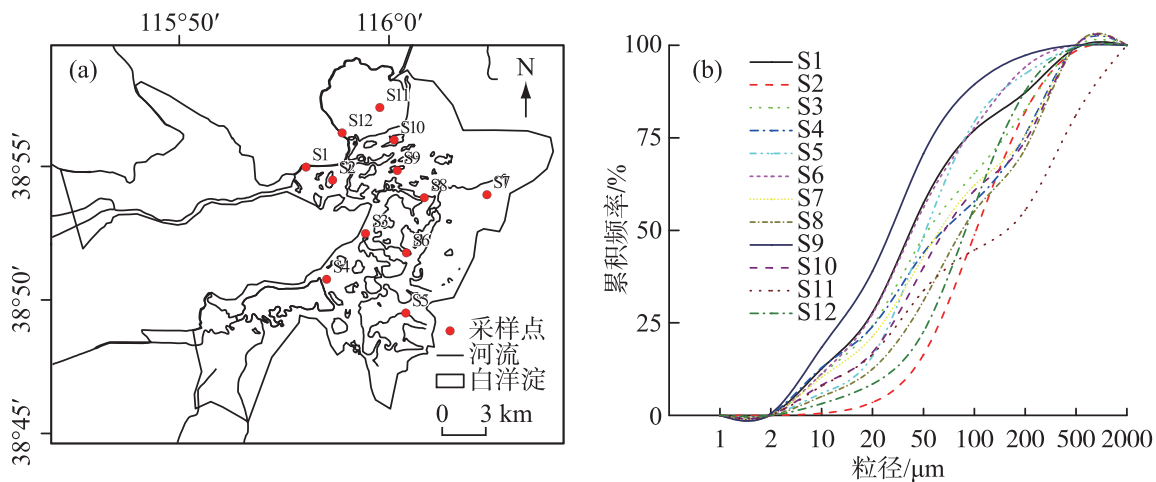

图 1 白洋淀采样点分布 (a), 不同采样点的沉积物颗粒级配曲线 (b)

Fig.1 Distribution of sampling sites in Lake Baiyangdian(a), accumulative percentage of sediment size of different sampling sites ( b )

本试验在长 $24 \mathrm{~m}$ 、宽 $38 \mathrm{~cm}$ 、高 $60 \mathrm{~cm}$ 的玻璃水槽中进行 (图 2). 为更好的阐述植被作用下近床面的沉 
表 1 波浪水槽试验参数设置

Tab.1 Wave and vegetation parameters

\begin{tabular}{|c|c|c|c|c|}
\hline $\begin{array}{l}\text { 植被 } \\
\text { 情景 }\end{array}$ & $\begin{array}{l}\text { 直径 } \\
d / \mathrm{cm}\end{array}$ & $\begin{array}{l}\text { 密度 } m / \\
\left(\text { 根 } / \mathrm{m}^{2}\right)\end{array}$ & $\begin{array}{c}\text { 植被固相 } \\
\text { 体积分数 } \phi\end{array}$ & $\begin{array}{c}\text { 植被间距 } \\
S / \mathrm{cm}\end{array}$ \\
\hline CO & 光滑床面 & 0 & 0 & - \\
\hline $\mathrm{C} 1$ & 0.32 & 201 & 0.002 & 7.1 \\
\hline $\mathrm{C} 2$ & & 398 & 0.003 & 5.0 \\
\hline $\mathrm{C} 3$ & & 1131 & 0.009 & 3.0 \\
\hline $\mathrm{C} 4$ & & 2121 & 0.017 & 2.2 \\
\hline C5 & 0.64 & 102 & 0.003 & 9.9 \\
\hline C6 & & 530 & 0.017 & 4.3 \\
\hline C7 & & 1033 & 0.033 & 3.1 \\
\hline C8 & & 1550 & 0.050 & 2.5 \\
\hline C9 & 1.26 & 136 & 0.017 & 8.6 \\
\hline C10 & & 265 & 0.033 & 6.1 \\
\hline C11 & & 397 & 0.050 & 5.0 \\
\hline C12 & & 795 & 0.099 & 3.5 \\
\hline
\end{tabular}

积物再悬浮过程, 同时防止流速测量仪和浓度测量 仪对近床面波速和紊流结果的影响, 选取长 $7 \mathrm{~cm}$ 直 径分别为 $0.32 、 0.64 、 1.26 \mathrm{~cm}$ 的模型植被 (圆木棒) 用 于水槽试验, 以模拟浅水湖泊中的芦苇 $(d=0.77 \pm$ $0.15 \mathrm{~cm}$ )、滨海湿地中的红树林 (根茎直径粗至 $2 \mathrm{~cm}^{[22]}$ ), 以及拥有此长度与直径刚性叶鞘的水生植 被. 研究结果表明, 不同波浪条件下, 淹没刚性植被 茎干产生的紊动能 $(T K E)$ 明显高于光滑床面, 淹没 植被上方无植被区域的紊动能与光滑床面近似, 植 被顶端并未出现紊动能峰值 ${ }^{[13]}$. 因此, $7 \mathrm{~cm}$ 的植被 高度足以反映植被茎干紊流特性与沉积物再悬浮过 程, 同时兼顾测量仪器位置和测量效果. 植被密度 $m=102 \sim 2121$ 根 $/ \mathrm{m}^{2}$, 固体体积分数 $\phi=0.002 \sim 0.099$ (表 1), 模型植被以交错排列方式插人长 $2 \mathrm{~m}$ 、宽 34 $\mathrm{cm}$ 的 PVC 底板中, 将底板置于水槽中间位置, 相邻 植被平均间距 $S=\sqrt{1 / m}$. 以中值粒径 $D_{50}=0.085 \pm$ $0.015 \mathrm{~cm}$ 的模型沙模拟白洋淀沉积物在不同植被情

景下的沉积物再悬浮规律, 每组试验之前在植被前端下游 $60 \mathrm{~cm}$ 处铺设长为 $100 \mathrm{~cm}$ 、厚约 $0.5 \pm 0.2 \mathrm{~cm}$ 的沉 积物层,如图 2 所示.

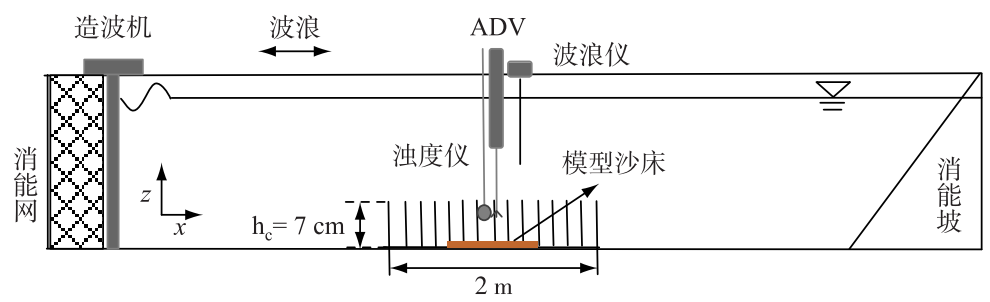

图 2 波浪水槽以及植被铺设示意

Fig.2 Schematic of wave channel and vegetation experiments

水槽前端通过液压驱动的垂直桨叶产生规则波浪, 通过计算机程序控制波的振幅和频率, 水槽末端设 有 $1: 5$ 的消能缓坡. 试验平均水深 $h=40 \mathrm{~cm}$, 波浪周期 $T=2 \mathrm{~s}$, 振幅 $a_{w}=1.5 \sim 3.7 \mathrm{~cm}$, 所产生的波速为 $4 \sim 20$ $\mathrm{cm} / \mathrm{s}$. 波浪位移 $\eta(t)$ 用频率为 $1000 \mathrm{~Hz}$ 的电阻型波浪仪测定, 波浪仪位于植被中部横向中间位置 (图 2), 根 据测量水面位移的均方根估算波幅, $a_{w}=\sqrt{2} \eta_{r m s}$. 运用 3D 声学多普勒测速仪 (ADV, Nortek Vectrino) 以 200 $\mathrm{Hz}$ 的采样频率测量 $x$ (纵向)、 $y$ (横向) 和 $z$ (垂直) 3 个方向的瞬时流速 $(u, v, w)$, 流速仪位于植被冠层中间 位置. 流速测量点为沙床上方 $1.3 \mathrm{~cm}$ 水深处, 每个流速情景下连续测量流速 $6 \mathrm{~min}$, 运用加速度阈值法去除 速度数据噪点 ${ }^{[23]}$.

波浪的瞬时速度 $u(t)$ 可分解为时均流速 $U_{c}$ 、震荡的波浪流速 $U_{w}(t)$ 和紊动流速 $u^{\prime}(t)$ （以 $u$ 为例, 对于 $v$ 和 $w$ 相同)：

$$
u(t)=U_{c}+U_{w}(t)+u^{\prime}(t)
$$

运用相位平均的方法, 将不同周期内同一相位的瞬时流速进行时间平均, 即可得到相位平均的流速 $\widetilde{u}(\varphi)$, 时均流速则可表示为相位平均流速在一个波浪周期内的积分平均 $\left(U_{c}=\int_{0}^{2 \pi} \widetilde{u}(\varphi) \mathrm{d} \varphi / 2 \pi\right)$, 波浪流速 则用相位平均后流速值的均方根表示:

$$
u_{r m s}=\sqrt{\frac{1}{2 \pi} \int_{0}^{2 \pi}\left[\tilde{u}(\varphi)-U_{c}\right]^{2} \mathrm{~d} \varphi}
$$


震荡的波浪速度也称轨道波速 $\left(U_{w}\right)$, 是波浪周期内相位平均流速的最大值, 可表示为 $U_{w}=2 \sqrt{u_{m s}}$. 紊动流速定义为瞬时流速 $u(t)$ 与相位平均流速值 $\widetilde{u}(\varphi)$ 之间的偏差, 每个相位内的均方根波动 $\left(u_{r m s}, v_{r m s}\right.$, $\left.w_{r m s}\right)$ 用于估计该相位内的紊动能,表示为:

$$
T K E=\left(u_{r m s}^{2}+v_{r m s}^{2}+w_{r m s}^{2}\right) / 2
$$

本文以 TKE 表示波周期内所有相位的时间平均的实测紊动能, 以 $k_{t}$ 表示植被紊流模型的计算紊动能. 运用光学后向散射浊度仪 (OBS, Seapoint Sensors Inc.) 测量悬浮颗粒浓度, 将其放置于植被层纵向和横向中 间位置, 浊度仪探头位于沙床上方 $5 \mathrm{~cm}$ 处, 探头朝下. 每个波浪情景下, 浊度仪以 $20 \mathrm{~Hz}$ 的采样频率连续采 集 $10 \mathrm{~min}$, 将电压输出结果转换为悬浮颗粒浓度.

\section{3 结果与讨论}

\section{1 植被紊动能}

对于单向流, 淹没植被层顶部阻力不连续, 通过剪切层不稳定性产生紊流相干结构, 并在冠层顶部产生 紊流峰值 ${ }^{[24]}$. 相比较而言, 在波浪情景下, 只有长波 $(T>12 \mathrm{~s})$ 流经密度较大的植被层时会在冠层顶部产生 剪切层不稳定性 ${ }^{[25]}$. 本研究所采用 $T=2 \mathrm{~s}$ 的波浪情景未产生紊流相干结构, 故仅考虑植被茎干产生的紊流.

在沙床以上 $1.3 \mathrm{~cm}$ 处的测量结果表明, 紊动能是波浪流速的平方 $\left(u_{r m s}^{2}\right)$ 和植被固相体积分数 $(\phi)$ 的函 数, 不同密度和直径的植被产生的紊动能随 $u_{r m s}^{2}$ 的增加而增加, $\phi$ 表示植被密度和直径对紊流的影响, 如图 3 所示. TKE 随 $\phi$ 的增加呈增加趋势, 对比不同直径同一 $\phi$ 的植被所产生的 TKE 发现, 尽管在植被密度更大的 情景下直径对 $T K E$ 的影响更大, 但整体上 $\phi$ 主导紊流的产生, 如图 3 中的 $\phi=0.05$ 和 0.099 . 对于所有具有 不同的 $\phi$ 和 $d$ 的植被情景而言, TKE 与 $u_{r m s}^{2}$ 呈正线性关系, 在波浪流速 $u_{r m s}$ 相对较低的情景下, 即使在较大的 直径范围内也会产生较小的 TKE. 图 3 中的线性关系也验证了公式 (4) 可以合理地描述植被对 $T K E$ 的影响.
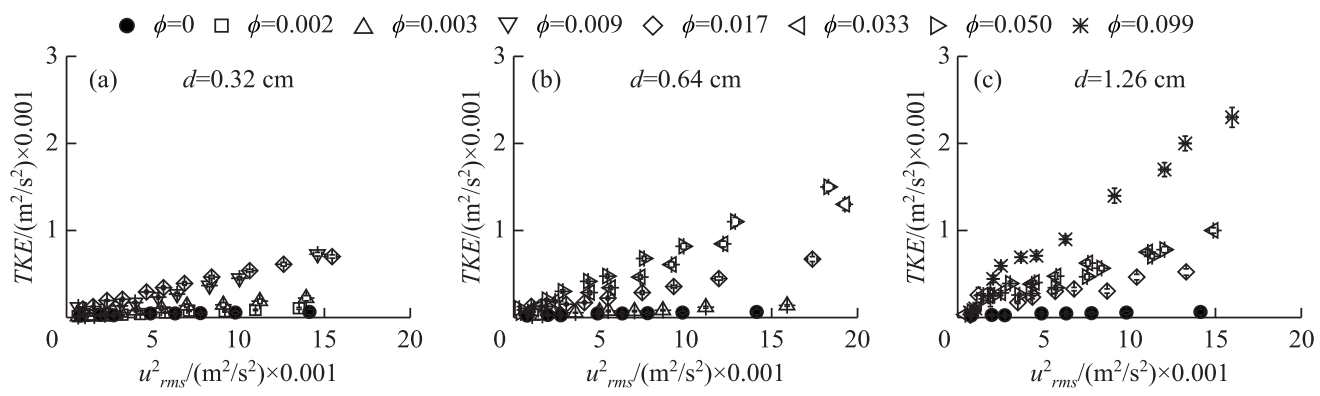

图 3 不同植被情景下的近床面紊动能 TKE 和 $u_{r m s}^{2}$ 的关系

Fig.3 Relationship between measured near-bed turbulent kinetic energy and $u_{r m s}^{2}$ within vegetation canopy

对于单一直径的植被 $(d=0.32 、 0.64$ 或 $1.26 \mathrm{~cm}$ ), 植被茎干 $\phi$ 越大所产生的 $T K E$ 越大 (图 4). 对于相同 的 $\phi$, 直径仍然影响 TKE, 尤其是对于直径较大的植被, 如 $\phi=0.017$ 和 0.05 , 相同 $\phi$ 不同直径所产生的 TKE 差异更大. 在图 $4 \mathrm{~b}, \mathrm{c}$ 中, 植被固相体积分数 $\phi=0.017$ 的 3 种直径的植被层产生的 $T K E$ 相近, 但当 $\phi=$ 0.05 时,直径 $d=0.64 \mathrm{~cm}$ (图 4b) 和 $d=1.26 \mathrm{~cm}$ (图 4c) 的植被层所产生的 TKE 则存在明显差异. 此外, 当波 浪轨道运动半径与植被间距的比值 $A_{w} / S$ 越大时 $\left(A_{w}=U_{w} T / 2 \pi\right)$, 刚性植被茎干所产生的紊动能越大, 尤其 是当 $A_{w} / S \geqslant 1$ 时, 植被茎干产生的紊流可以传播到整个植被层, 前人对沉水植被刚性叶鞘的研究也揭示了 同样的结果 ${ }^{[11]}$. 而对于 $A_{w} / S<0.5$, 本研究中茎干产生的紊流与前人研究中 ${ }^{[11]}$ 光滑床面产生的 $T K E$ 相当, Zhang 等的结果是基于 $\phi=0.011 \sim 0.051^{[11]}$, 该植被 $\phi$ 范围小于本研究中的 $\phi$ 范围 $(\phi=0.0016 \sim 0.099)$. 本 研究中的 $A_{w} / S=0.16 \sim 3.21$ (图 4), 当 $A_{w} / S>1$ 时, 植被阻力主导水流, 导致冠层内的波速小于冠层外; 相 反, 波的传播与主导惯性力有关, 在 $A_{w} / S<1$ 的情况下冠层内的速度会变大 ${ }^{[13]}$, 这表明植被所产生的 $T K E$ 将根据 $A_{w} / S$ 的不同分为两部分.

为确定植被紊流模型中紊流的比例因子 $\delta^{2}$, 将实测 $T K E$ 与紊流模型预测值(公式 (4) 等式右边第一项) 

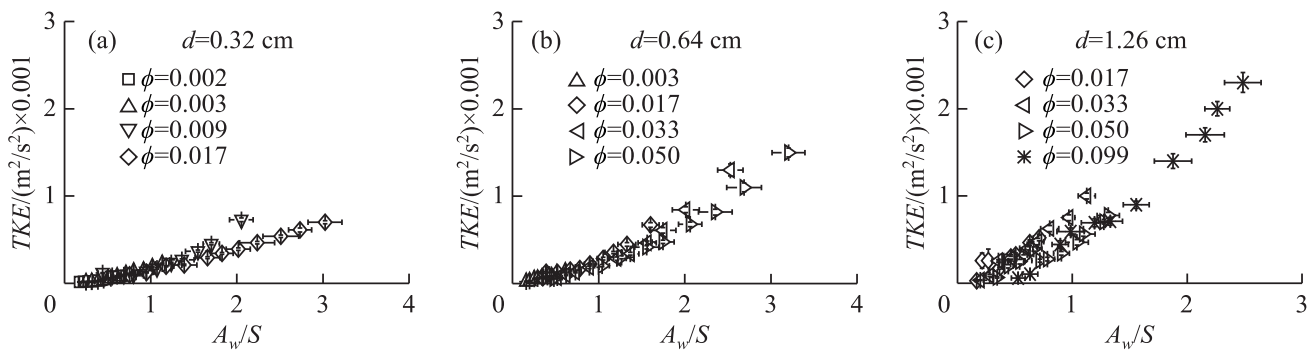

图 4 实测植被层近床面紊动能 $T K E$ 与 $A_{w} / S$ 的关系

Fig.4 Relationship between measured near-bed $T K E$ and $A_{w} / S$

进行比较, 比较结果如图 5 所示. 从图 3 可知, $\phi=0.002$ 和 0.003 的植被层具有与光滑床面 $(\phi=0)$ 相近的 紊流水平, 因此图 5 中不考虑 $\phi=0.002$ 和 0.003 的植被产生的紊动能. 植被阻力系数 $C_{D}$ 根据 Keulegan-Carpenter 数 ${ }^{[26]}$ (简称 $K C$ 数, $K C=U_{w} T / d$ ) 估算, 得到 3 种直径的植被阻力系数 $C_{D}=1.5 \pm 0.3$. 实测紊动能与公式 (4) 中植被紊动能的预测值的线性拟合的斜率为 $\delta=0.78 \pm 0.02(95 \% \mathrm{CI})$, 即紊流比例因子 $\delta^{2}=0.61 \pm 0.02$.

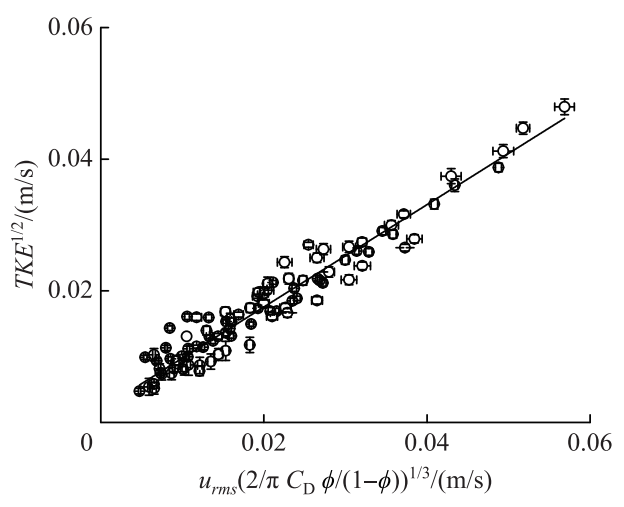

图 5 近床面紊动能实测值与植被紊流模型计算值对比

Fig.5 Comparison of measured near-bed TKE with predicted vegetation-generated turbulence

\section{2 沉积物再悬浮浓度与临界速度}

沉积物再悬浮浓度受植被固相体积分数和波浪条件共同影响,用 $\phi$ 表示直径和密度的影响 (图 6). 当 悬浮颗粒平均浓度高于 OBS 仪器噪声水平时, 则认为沉积物开始悬浮, 此时的波速为沉积物再悬浮的临界 波速, 仪器噪声水平即是不同植被情景下静水时测量浓度的平均值, 为 $7 \mathrm{mg} / \mathrm{L}$. 本研究所用的模型沙的沉降 时间为沙子在 OBS 测量深度 $\left(h^{\prime}=5 \mathrm{~cm}\right)$ 上的标准化沉降速度, 即沉降速度 $w_{s}=0.4 \mathrm{~cm} / \mathrm{s}$, 沉降时间 $h^{\prime} / w_{s}=$ $1.25 \mathrm{~s}$. 对于已经悬浮的颗粒沙, 沉降时间小于波浪周期 $(T=2 \mathrm{~s})$, 表明部分颗粒在较小的波浪条件下沉降.

除密度较小的植被情景 $(\phi=0.002$ 和 0.003 ) 外,其余植被情景下沉积物再悬浮的临界波速小于光滑床 面的临界速度值,一旦沉积物重新悬浮, 相同的波速下, 悬浮浓度随植被固相体积分数 $\phi$ 的增加而增加 ( 图 6). 该结果表明, 在大多数植被情景下, 与光滑床面相比, 植被的存在增强了沉积物再悬浮. 同样的结果也曾 被报道: 悬浮浓度受到固体体积分数 $\phi$ 的影响, 且具有较大 $\phi$ 的植被情景下沉积物再悬浮时间更早 ${ }^{[27]}$. 在 直径更大 $(d=2 \mathrm{~cm})$ 的模型植被层中, 单向水流下的沉积物再悬浮浓度随 $\phi$ 的增加而增加 ${ }^{[28]}$. Tinoco 和 Co$\mathrm{co}^{[12]}$ 也阐明了植被层中的沉积物再悬浮浓度随 $\phi(\phi=0 、 0.008 、 0.047 、 0.079)$ 的增加而增加这一类似结论, 在波浪扰动下,植被产生的紊动能比床面切应力更能促进沉积物再悬浮.

所有直径的植被层中沉积物再悬浮临界速度 $u_{r m s, c}$ 随固相体积分数 $\phi$ 的增加而降低 (图 7), 由此可知, $\phi$ 对沉积物再悬浮的重要性高于植被直径 $d$, 这一试验结果与植被茎干产生的紊流控制沉积物再悬浮理论一 

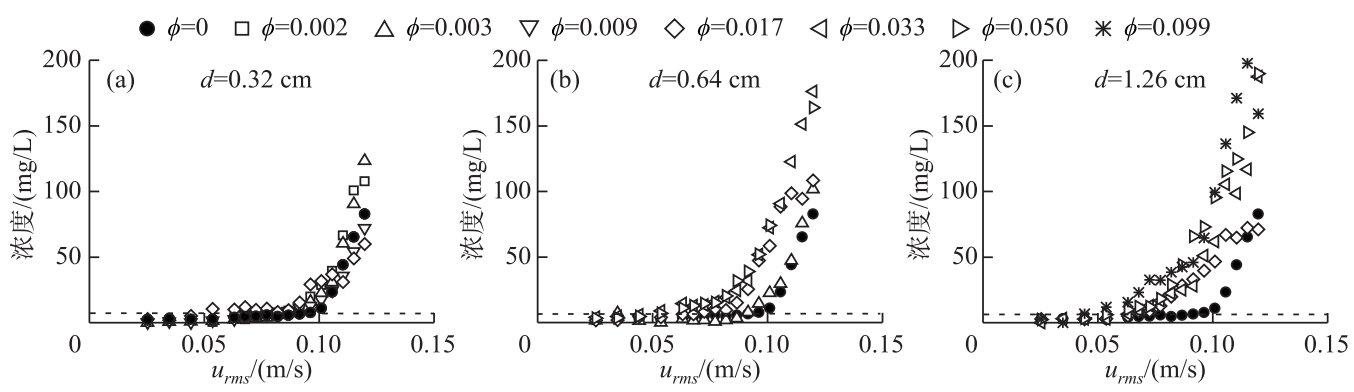

图 6 沉积物再悬浮浓度与 $u_{r m s}$ 之间的关系

Fig.6 Suspended sediment concentration as a function of wave velocity $\left(u_{r m s}\right)$

致(公式 (4) 右边第一项). 因此, 本研究将圆柱形植被层影响下的沉积物再悬浮临界速度的观测范围限定 在植被直径 $d=0.32 \sim 1.26 \mathrm{~cm}$.

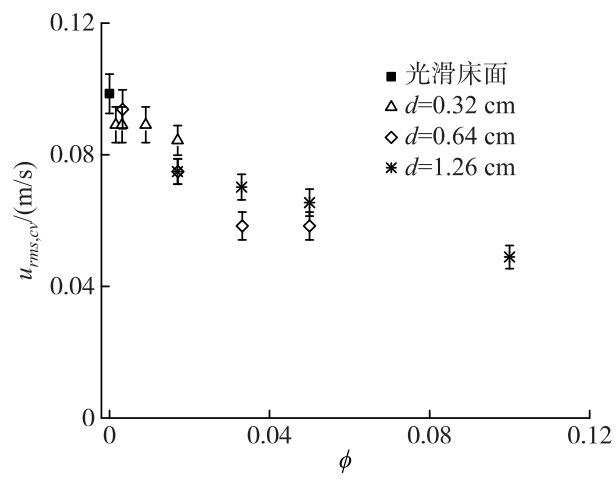

图 7 不同直径植被下沉积物再悬浮临界速度与植被固相体积分数

Fig.7 Critical velocity for wave-driven resuspension within arrays of circular cylinders of diameter $d$ versus solid volume fraction $\phi$

光滑床面所测量的沉积物颗粒 $\left(D_{50}=85 \pm 21 \mu \mathrm{m}\right)$ 再悬浮的临界速度均值为 $u_{r m s, c o}=9.1 \mathrm{~cm} / \mathrm{s}$, 则光滑床 面沉积物再悬浮临界状态的最大波速即是轨道波速 $U_{w}=12.8 \pm 0.9 \mathrm{~cm} / \mathrm{s}$. Shields 数 $\left(\theta=\tau_{b} /\left(\rho_{s}-\rho\right) g D_{50}\right)$ 与颗粒粒径相关, 可用于确定颗粒运动的临界状态, 当 $\theta$ 值大于临界 $\theta$ 时, 可认为是沉积物再悬浮的初始状 态 ${ }^{[29]}$. 由此可知, 小颗粒的再悬浮临界速度较低. 遵循 Yang 等 ${ }^{[17]}$ 与 Tinoco 和 $\mathrm{Coco}^{[12]}$ 的结论, 植被产生的紊 流是沉积物颗粒起动和再悬浮的主要驱动因素. 图 8 比较了悬浮颗粒浓度和近床面的紊流水平, 在大多数 直径情景下 $(d=0.32 、 0.64 、 1.26 \mathrm{~cm})$, 沉积物悬浮颗粒浓度在 $T K E \approx 3 \times 10^{-4} \mathrm{~m}^{2} / \mathrm{s}^{2}$ 时高于测量仪器噪声水平 7 $\mathrm{mg} / \mathrm{L}$ (图 8 中的黑虚线). 已有研究发现在 $d=2 \mathrm{~cm}$ 的随机植被分布格局中, 波浪扰动的植被情景下, 沉积 物再悬浮的植被紊动能阈值 $T K E \approx 6 \times 10^{-4} \mathrm{~m}^{2} / \mathrm{s}^{2[12]}$. 此 TKE 阈值略大于本研究中的阈值 $\left(T K E \approx 3 \times 10^{-4}\right.$ $\left.\mathrm{m}^{2} / \mathrm{s}^{2}\right)$, 其原因是该文献研究使用的沉积物颗粒 $\left(D_{50}=280 \mu \mathrm{m}\right)$ 稍大于本研究 $\left(D_{50}=85 \pm 21 \mu \mathrm{m}\right)$.

所有试验情景中有 4 种情景的沉积物再悬浮临界紊动能低于前文所述的临界水平 $\left(3 \times 10^{-4} \mathrm{~m}^{2} / \mathrm{s}^{2}\right)$, 分 别是光滑床面 $(\phi=0)$ 、直径 $d=0.32 \mathrm{~cm}$ 的 $\phi=0.002$ 和 $\phi=0.003$ ( 图 8a), 以及直径 $d=0.64 \mathrm{~cm}$ 的 $\phi=0.003$ (图 8b). 可能的原因是, 对于光滑床面和 $\phi$ 值较低的情景, 床面产生的紊流是近床面总紊动能的最主要来 源. 因此, 在推求沉积物再悬浮的临界紊动能时, 不考虑上述 4 种情景的 TKE. 根据其余植被情景得到沉积 物再悬浮的临界紊动能 $k_{t, c}=2.6 \pm 1.0\left(\times 10^{-4} \mathrm{~m}^{2} / \mathrm{s}^{2}\right)$, 其中 $k_{t, c}$ 的不确定性为不同植被情景的标准偏差.

根据公式 (4) 的理论, 特定沉积物颗粒的再悬浮临界紊动能为常数. 由前文可知光滑床面沉积物再悬浮 临界轨道波速 $U_{w, c o}=12.8 \pm 0.9 \mathrm{~cm} / \mathrm{s}$, 由此可得沙床粗糙度系数 $C_{b, w}=k_{t, c} / U_{w, c o}^{2}=0.02 \pm 0.01$. 从图 5 可知 $\delta^{2}=$ 

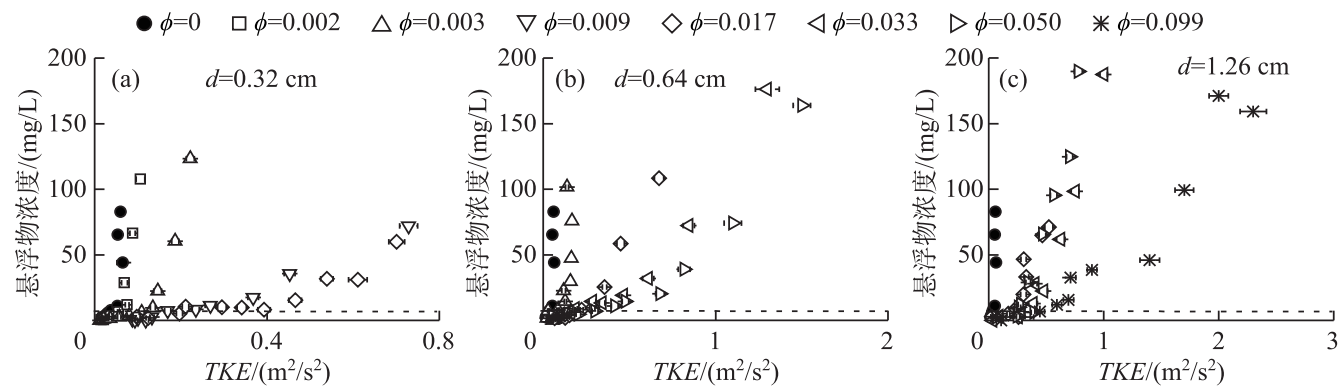

图 8 不同植被情景下沉积物悬浮物浓度与近床面紊动能之间的关系

Fig. 8 Suspended sediment concentration as a function of near-bed turbulent kinetic energy for cylinder diameters (a) $0.32 \mathrm{~cm}$, (b) $0.64 \mathrm{~cm}$, and (c) $1.26 \mathrm{~cm}$

$0.61 \pm 0.02$, 将 $\delta^{2}$ 和 $C_{b, w}$ 代人公式 (4) 中, 得到植被影响下沉积物再悬浮的临界波速 $U_{w, c}$. 将 $C_{D}=1.5 \pm 0.3$ 代人 公式 (5), 得紊动能预测值与实测值的相互关系 (图 9). 图 9a 中 $U_{w, c} / U_{w, c o}$ 的预测值与实测值误差在 $20 \%$ 以 内,该结果证实公式 (5) 的适用范围为固相体积分数小于 $10 \%(\phi<0.1)$ 的植被茎干紊流. 运用 Tinoco and Co$\mathrm{co}^{[12]}$ 的水槽试验数据 (淹没刚性植被直径 $d=2 \mathrm{~cm}$, 植被高度 $h_{c}=21 \mathrm{~cm}$, 密度 $m=25 \sim 250$ 根 $/ \mathrm{m}^{2}, \phi=0.008 \sim$ 0.079 , 沙床粒径 $D_{50}=280 \mu \mathrm{m}$ ) 对本模型进行验证, 结果显示 Tinoco and $\mathrm{Coco}^{[12]}$ 的实测数据在本模型的预测 范围内 (图 9b), 表明本模型的沉积物颗粒适用范围从 $D_{50}=85 \mu \mathrm{m}$ 扩展至 $D_{50}=280 \mu \mathrm{m}$, 植被茎干直径从 $d=$ $0.32 \sim 1.26 \mathrm{~cm}$ 扩展至 $d=2 \mathrm{~cm}$.
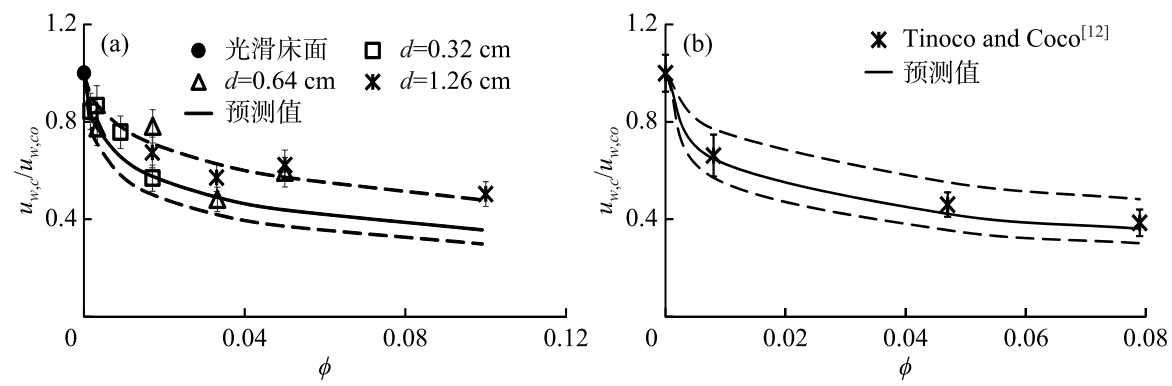

图 9 沉积物再悬浮临界波速实测值与模型预测值

Fig.9 Comparison of predicted and measured critical wave velocity

本研究仅考虑具有单个周期 $(T=2 \mathrm{~s})$ 的波, 对应野外的风生波, 可近似于例如大型浅水湖泊太湖风生浪 的波浪周期 $T=1.2 \sim 1.8 \mathrm{~s}$ 和波高 $(2 \mathrm{~cm} \text { 和 } 10 \mathrm{~cm})^{[30]}$. 但本文提出的植被紊流模型可能不适用于流经植被 层的长波, 长波会在植被冠层顶部产生剪切紊流, 并有助于再悬浮 ${ }^{[25]}$, 如受海洋重力波等长波影响的潮滩植 被. 此外, 本研究所提出的波浪驱动下刚性植被层中沉积物再悬浮模型, 可与植被影响下的波浪衰减模型相 结合, 综合考虑植被层对波浪的衰减和沉积物再悬浮的抑制作用, 以及植被茎干产生的紊流对沉积物再悬 浮的促进作用, 以此得到实际野外波浪扰动下植被对沉积物的综合影响, 并得出基于沉积物沉降或再悬浮 需求的适宜植被面积、植被密度和高度等分布特征, 为浅水湖泊和滨海湿地生态修复与保护提供科学指导 与关键参数.

\section{4 结论}

本研究通过室内水槽试验探究了刚性模型植被对波浪驱动的沉积物再悬浮的影响, 植被层设定了 3 种 直径 $(d=0.32 、 0.64$ 和 $1.26 \mathrm{~cm}) 、 4$ 种固相体积分数 $(\phi)$ 和不同的波浪条件. 结果表明: 1$)$ 植被改变波速结构 并产生紊动能 $(T K E)$, 含植被近床面的总 $T K E$ 由植被产生的紊流和床面粗粘度产生的紊流共同决定;2) 植 
被层近床面 TKE 随 $\phi$ 增大而增大,与波速均方根的平方 $\left(u_{r m s}^{2}\right)$ 呈线性关系;3) $\phi>0.003$ 的植被茎干产生的紊 流可局部增强沉积物再悬浮, 当近床面总 $T K E$ 大于 $2.6 \pm 1.0\left(\times 10^{-4} \mathrm{~m}^{2} / \mathrm{s}^{2}\right)$ 时, 沉积物开始悬浮, 且 $\phi$ 越大沉 积物悬浮越早; 3 ) 构建并验证了公式 (4) 作为预测波浪驱动的沉积物再悬浮的植被紊流模型, 基于植被近床 面总 TKE 值确定了沉积物再悬浮的波速國值(公式(5)), 用于预测沉积物再悬浮的临界波速. 该植被紊流 模型适用于波浪驱动下沉积物颗粒粒径 $D_{50}=85 \sim 280 \mu \mathrm{m}$, 植被茎干直径 $d=0.32 \sim 2 \mathrm{~cm}$ 的刚性植被层沉积 物再悬浮过程.

\section{5 参考文献}

[ 1 ] Gao P, Zhou ZZ, Ma SY et al. Vegetation distribution pattern and community succession in the transition from macrophyteto phytoplankton-dominated state in shallow lakes, a case study of Lake Caizi in Anhui Province. J Lake Sci, 2011, 23 (1)：13-20. DOI: 10.18307/2011.0103. [高攀, 周忠泽, 马淑勇等. 浅水湖泊植被分布格局及草-藻型生态系统转 化过程中植物群落演替特征: 安徽菜子湖案例. 湖泊科学, 2011, 23(1): 13-20.]

[ 2 ] Feng JX, Huang Q, Chen H et al. Effects of Spartina alterniflora invasion on benthic faunal community in saltmarsh and mangrove wetland. Chinese Journal of Ecology, 2018, 37(3) : 943-951. DOI: 10.13292/j.1000-4890.201803.012. [冯建 祥, 黄茜, 陈卉等. 互花米草人侵对盐沼和红树林滨海湿地底栖动物群落的影响. 生态学杂志, 2018, 37 (3) : 943-951.]

[ 3 ] Yi Y, Tang C, Yi T et al. Health risk assessment of heavy metals in fish and accumulation patterns in food web in the upper Yangtze River, China. Ecotoxicology and Environmental Safety, 2017, 145: 295-302. DOI: 10.1016/j.ecoenv.2017. 07.022 .

[ 4 ] Shields FD, Bowie AJ, Cooper CM. Control of streambank erosion due to bed degradation with vegetation and structure. Journal of the American Water Resources Association, 1995, 31(3) : 475-489. DOI : 10.1111/j.1752-1688.1995.tb04035.x

[ 5 ] Zhou WW, Shao DD, Sun T et al. Simulating plant exposure to long-term shallow water waves-system design and trial experiments. Journal of Beijing Normal University: Natural Science, 2018, 54(1): 113-117. [周薇薇, 邵冬冬, 孙涛等. 植物长期暴露于浅水波环境的模拟装置研制与试验. 北京师范大学学报：自然科学版, 2018, 54(1): 113-117.]

[ 6 ] Qin B, Hu W, Gao G et al. Dynamics of sediment resuspension and the conceptual schema of nutrient release in the large shallow Lake Taihu, China. Chinese Science Bulletin, 2004, 49(1) : 54-64. DOI: 10.1007/BF02901743.

[ 7 ] Bradley K, Houser C. Relative velocity of seagrass blades: Implications for wave attenuation in low-energy environments. Journal of Geophysical Research, 2009, 114(F1). DOI: 10.1029/2007JF000951.

[ 8 ] Cao HJ, Feng WB. Study on the characteristic of wave dissipation by artificial flexible vegetation field. The Ocean Engineering, 2014, 32(3) : 36-44. [ 曹海锦, 冯卫兵. 人工柔性植被场中波浪衰减特性研究. 海洋工程, 2014, 32(3): 36-44.]

[ 9 ] Hu XZ, Liu Q, Li YJ. The controlling of plants rebuild engineering on sediment resuspension and nutrient salts release in Fubao Bay of the Dianchi Lake. China Environmental Science, 2012, 32(7): 1288-1292. [胡小贞, 刘倩, 李英杰. 滇池 福保湾植被重建对底泥再悬浮及营养盐释放的控制. 中国环境科学, 2012, 32(7) : 1288-1292.]

[10] Pujol D, Casamitjana X, Serra T et al. Canopy-scale turbulence under oscillatory flow. Continental Shelf Research, 2013, 66: 9-18. DOI: $10.1016 /$ j.csr.2013.06.012.

[11] Zhang Y, Tang C, Nepf H. Turbulent kinetic energy in submerged model canopies under oscillatory flow. Water Resources Research, 2018, 54(3) : 1734-1750. DOI: 10.1002/2017WR021732.

[12] Tinoco RO, Coco G. Turbulence as the main driver of resuspension in oscillatory flow through vegetation. Journal of Geophysical Research: Earth Surface, 2018, 123(5) : 891-904. DOI: 10.1002/2017JF004504.

[13] Ros À, Colomer J, Serra T et al. Experimental observations on sediment resuspension within submerged model canopies under oscillatory flow. Continental Shelf Research, 2014, 91: 220-231. DOI: 10.1016/j.csr.2014.10.004.

[14] Hansen JCR, Reidenbach MA. Wave and tidally driven flows in eelgrass beds and their effect on sediment suspension. Marine Ecology Progress Series, 2012, 448: 271-287. DOI: 10.3354/meps09225.

[15] Luhar M, Infantes E, Nepf H. Seagrass blade motion under waves and its impact on wave decay. Journal of Geophysical Research: Ocean, 2017, 122(5) : 3736-3752. DOI: 10.1002/2017JC012731.

[16] Tanino Y, Nepf HM. Lateral dispersion in random cylinder arrays at high Reynolds number. Journal of Fluid Mechanics, 
2008, 600: 339-371. DOI: $10.1017 /$ S0022112008000505.

[17] Yang JQ, Chung H, Nepf HM. The onset of sediment transport in vegetated channels predicted by turbulent kinetic energy. Geophysical Research Letters, 2016, 43(21) : 11261-11268. DOI: 10.1002/2016GL071092.

[18] Stapleton K, Huntley D. Seabed stress determinations using the inertial dissipation method and the turbulent kinetic energy method. Earth Surface Processes and Landforms, 1995, 20(9) : 807-815. DOI: 10.1002/esp.3290200906.

[19] Sumer BM, Chua LHC, Cheng NS et al. Influence of turbulence on bed load sediment transport. Journal of Hydraulic Engineering, 2003, 129(8) : 585-596. DOI: 10.1061/(asce) 0733-9429(2003) 129:8( 585 ).

[20] Green MO, Coco G. Review of wave driven sediment resuspension and transport in estuaries. Reviews of Geophysics, 2014, 52 (1) : 77-117. DOI: 10.1002/2013RG000437.

[21] Xie R, Ji CH, Wang YP et al. Transporting experimental study of the bottom silt-clay particles under the lake wave movement. J Lake Sci, 2016, 28(3) : 669-675. DOI : 10.18307/2016.0324. [谢瑞, 姬昌辉, 王永平等. 波浪作用下湖泊底 泥的输沙试验研究. 湖泊科学, 2016, 28(3) : 669-675.]

[22] Norris BK, Mullarney JC, Bryan KR et al. The effect of pneumatophore density on turbulence: A field study in a Sonneratia-dominated mangrove forest. Vietnam, 2017, 147: 114-127. DOI: 10.1016/j.csr.2017.06.002.

[23] Goring DG, Nikora VI. Despiking acoustic Doppler velocimeter data. Journal of Hydraulic Engineering, 2002, 128( 1) : 117-126. DOI: 10.1061/( ASCE) 0733-9429(2002) 128:1(117).

[24] Nepf HM. Flow and transport in regions with aquatic vegetation. Annual Review of Fluid Mechanics, 2012, 44: 123-142. DOI: 10.1146/annurev-fluid-120710-101048.

[25] Ghisalberti M, Schlosser T. Vortex generation in oscillatory canopy flow. Journal of Geophysical Research: Ocean, 2013, 118(3) : 1534-1542. DOI: 10.1002/jgrc.20073.

[26] Keulegan GH, Carpenter LH. Forces on cylinders and plates in an oscillating fluid. Journal of Research of the National Bureau of Standards, 1958, 60(5) : 423-440. DOI: 10.6028/jres.060.043.

[27] MacVean LJ, Lacy JR. Interactions between waves, sediment, and turbulence on a shallow estuarine mudflat. Journal of Geophysical Research: Oceans, 2014, 119(3) : 1534-1553. DOI: 10.1002/2013JC009477.

[28] Tinoco RO, Coco G. A laboratory study on sediment resuspension within arrays of rigid cylinders. Advances in Water Resources, 2016, 92 : 1-9. DOI: 10.1016/j.advwatres.2016.04.003.

[29] Van Rijn LC ed. Principles of sediment transport in rivers, estuaries and coastal seas. Amsterdam: Aqua Publications, 1993.

[30] Ding Y, Sun L, Qin B et al. Characteristics of sediment resuspension in Lake Taihu, China: A wave flume study. Journal of Hydrology, 2018, 561 : 702-710. DOI: 10.1016/j.jhydrol.2018.04.026. 